J. Clin. Chem. Clin. Biochem.

Vol. 17, 1979, pp. 689-691

\title{
Influence of Indicating Enzyme Reaction on Apparent Creatine Kinase Activity
}

Creatine Kinase in Serum, VII.

By G. Szäsz†, Elfriede Kinne

Institute of Clinical Chemistry, University Medical School, Klinikstr. 32b, 6300 Gießen, Germany

\section{J. P. Colombo}

Central Chemical Laboratory, Inselspital, 3010 Bern, Switzerland

and

W. Gruber

Research Center Boehringer Mannheim GmbH, 8132 Tutzing, Germany

(Received September 3, 1979)

Summary: Comparison of the indicative systems yeast glucose-6-phosphate dehydrogenase/NADP ${ }^{+}$, leuconostoc glucose-6-phosphate dehydrogenase/NADP ${ }^{+}$and leuconostoc glucose-6-phosphate dehydrogenase/NAD ${ }^{+}$showed excellent correlation and no differences in apparent creatine kinase activity with the two methods using NADP ${ }^{+}$. By using $\mathrm{NAD}^{+}$with the leuconostoc enzyme relative recovery of apparent creatine kinase activity is lower due to interference of other serum constituents. The mean value of the relative differences versus methods using NADP $^{+}$was $5.8 \%$ in our experiments.

\section{Abhängigkeit der Kreatinkinase-Aktivität vom gewählten Indikator-System}

\section{Kreatinkinase-Aktivität im Serum, 7. Mitteilung}

Zusammenfassung: Der Vergleich der Indikator-Systeme Hefe-Glucose-6-phosphat-dehydrogenase/NADP ${ }^{+}$, Leuconostoc-Glucose-6-phosphat-dehydrogenase/NADP ${ }^{+}$und Leuconostoc-Glucose-6-phosphat-dehydrogenase/NAD ${ }^{+}$ zeigte für die zwei Indikator-Systeme mit $\mathrm{NADP}^{+}$eine vorzügliche Korrelation zwischen den Methoden und keine methodischen Differenzen bezüglich der Kreatinkinase-Aktivität. Bei Verwendung des Leuconostoc-Glucose-6phosphat-dehydrogenase/NAD ${ }^{+}$-Systems wird im Vergleich zum Hefe-Glucose-6-phosphat-dehydrogenase/NADP ${ }^{+}$. System eine geringere Kreatinkinașe-Aktivität gef̣unden. Dies wird auf den Einfluß anderer Serumbestandteile zurückgeführt. Nach unseren Ergebnissen findet im Mittel das NADP ${ }^{+}$-System 5,8\% mehr.

\section{Introduction}

In a series of publications Gábor Sżász (1-6) submitted detailed information on the background of the studies on creatine kinase activity determination in serum leading to common recommendations of some national societies for Clinical Chemistry. (7-11) When Gábor Szász died a cooperative study on the influence of different indicating systems was running in Gießen, Bern and Tutzing. The results reported here complete the series, together with an review on the reasons for the selection of $\mathrm{N}$-acetyl-cysteine as reactivating thiol to be published in Clinical Chemistry.

Coenzyme specificities of glucose-6-phosphate dehydrogenase preparations from yeast and leuconostoc species are different (12). The yeast enzyme is strongly specific for $\mathrm{NADP}^{+}$whereas the leuconostoc enzyme can use both $\mathrm{NADP}^{+}$and $\mathrm{NAD}^{+}$, the latter with lower affinity (13). As most dehydrogenases which might be present in human sera with higher activities are specific toward $\mathrm{NAD}^{+} / \mathrm{NADH}$, enhanced interference was expected sub- 
stituting $\mathrm{NADP}^{+}$by $\mathrm{NAD}^{+}$in creatine kinase assays $(14,15)$.

\section{Materials and Methods}

Adenosine diphosphate, adenosine monophosphate, diadenosine pentaphosphate, $\mathrm{N}$-acetyl-cysteine, creatine phosphate, glucose and hexokinase were dissolved to a common stock solution of $10 \%$ higher concentrations as compared to the recommended methods in imidazole buffer pH 6,7 containing $2 \mathrm{mmol} / 1$ EDTA. To each $90 \mathrm{ml}$ of this stock solution were added:

for method 1:

$0,438 \mathrm{~g} \mathrm{NADP}+$, disodium salt and $440 \mathrm{U}$ yeast glucose-6-phosphate dehydrogenase

for method 2:

$0,438 \mathrm{~g} \mathrm{NADP}+$ disodium salt and 440 U leuconostoc glucose6-phosphate dehydrogenase.

for method 3:

$0,354 \mathrm{~g} \mathrm{NAD}$, free acid and $440 \mathrm{U}$ leuconostoc glucose-6phosphate dehydrogenase.

These assay solutions were brought to $100 \mathrm{ml}$ by addition of water and used the same day for creatine kinase assays. All reagents were from Boehringer Mannheim GmbH. The samples were stored at $+4^{\circ} \mathrm{C}$ and assayed on the day of collection.

\section{Results}

65 samples with creatine kinase activity above $40 \mathrm{U} / 1$ were assayed with all 3 methods.

Figure 1 shows the excellent correlation of yeast glucose6-phosphate dehydrogenase/NADP ${ }^{+}$versus leuconostoc glucose-6-phosphate dehydrogenase/NADP ${ }^{+}$.

Figure 2 shows the relative differences of the methods as a function of creatine kinase activity obtained with method 1 (yeast glucose-6-phosphate dehydrogenase/ $\mathrm{NADP}^{+}$).

In table 1 the differences found are tabulated.

Figure 3 gives the correlation between yeast glucose-6phosphate dehydrogenase/NADP ${ }^{+}$versus leuconostoc

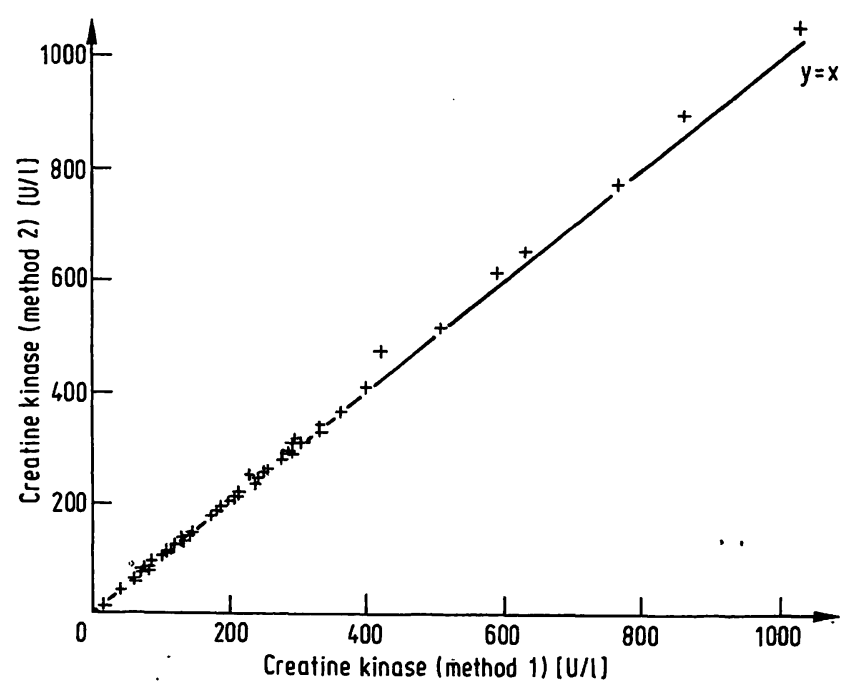

Fig. 1. Diagram of pairs of measured values.

Method 1: yeast glucose-6-phosphate dehydrogenase $1.73 \mathrm{kU} / 1$ assay volume. $\mathrm{NADP}^{+}: 2 \mathrm{mmol} / \mathrm{l}$. Method 2: leuconostoc glucose-6-phosphate dehydrogenase: $1.73 \mathrm{kU} / \mathrm{l}$ assay vomume. $\mathrm{NADP}^{+}: 2 \mathrm{mmol} / \mathrm{l}$.

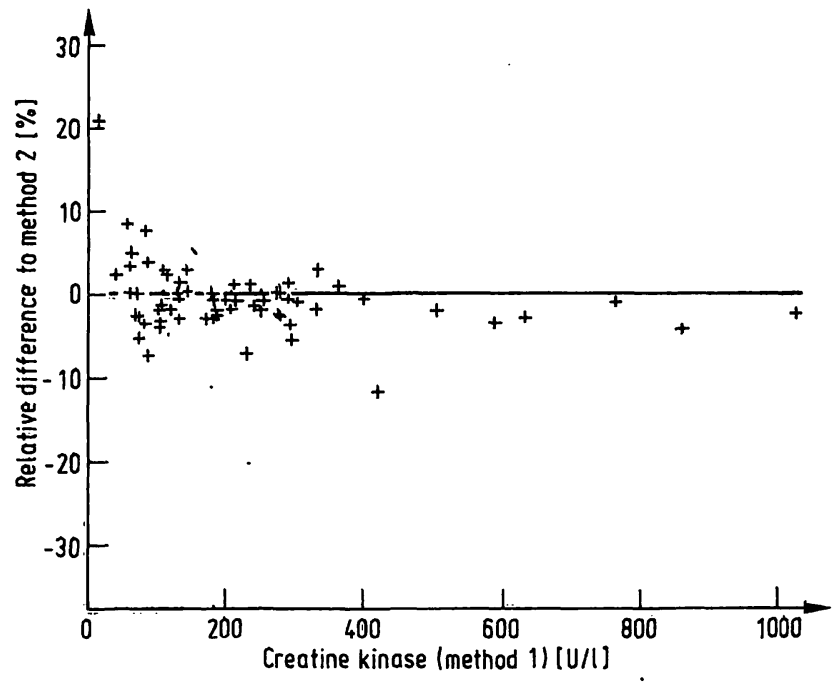

Fig. 2. Relative differences of methods.

(method 1 - method 2) $100 /$ method 1 as related to shown glucose-6-phosphate dehydrogenase activities found with method 1.

Method 1: yeast glucose-6-phosphate dehydrogenase $1.73 \mathrm{kU} / \mathrm{l}$ assay volume. $\mathrm{NADP}^{+}: 2 \mathrm{mmol} / \mathrm{l}$.

Method 2: leuconostoc glucose-6-phosphate dehydrogenase $1.73 \mathrm{kU} / 1$ assay volume. $\mathrm{NADP}^{+}: 2 \mathrm{mmol} / \mathrm{l}$.

Tab. 1. Differences found between method 1 and method 2 .

$\begin{aligned} & \text { Relative Differences } \\ & \text { of methods }\end{aligned}$
$\begin{aligned} & \text { Absolute differences } \\ & \text { of methods }\end{aligned}$
$\frac{\text { method } 1 \text {-method } 2) \cdot 100}{\text { method } 1}$

(method 1-method 2)

\begin{tabular}{lrr}
\hline Mean value & $-0,8 \%$ & $-4,3 \mathrm{U} / 1$ \\
Difference $_{\min .}$ & $-12,0 \%$ & $-51,0 \mathrm{U} / 1$ \\
Difference $_{\max .}$ & $20,7 \%$ & $9,5 \mathrm{U} / 1$ \\
\hline
\end{tabular}

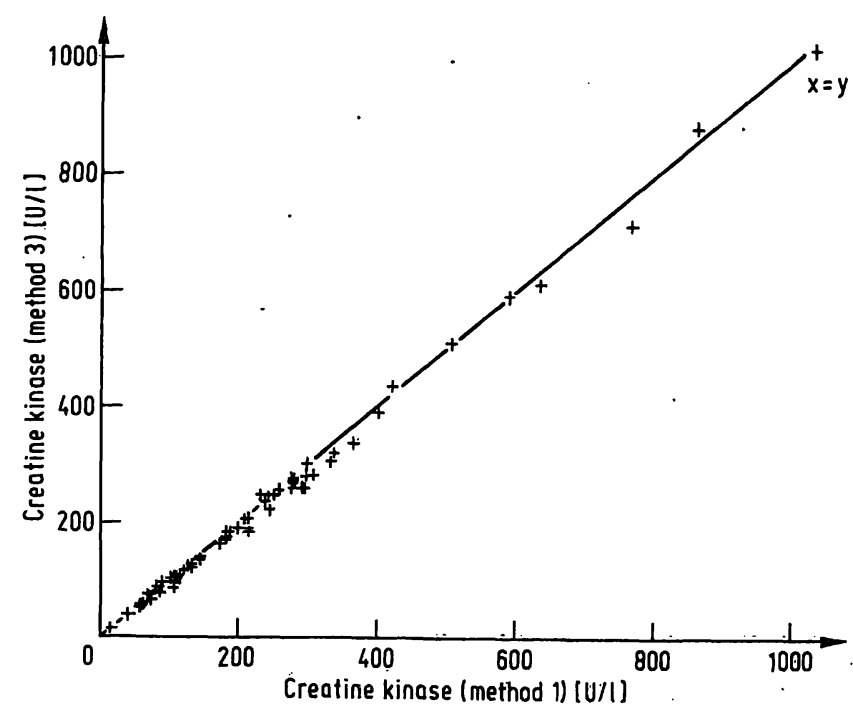

Fig. 3. Diagram of pairs of measured values.

Method 1: yeast glucose-6-phosphate dehydrogenase $1.73 \mathrm{kÜ} / 1$ assay volume. NAD $\overline{\mathrm{P}}^{+}: 2 \mathrm{mmol} / 1$. Method 3: leuconostoc glucose-6-phosphate dehydrogenase: $1.73 \mathrm{kU} / \mathrm{l}$ assay volume. $\mathrm{NAD}^{+}: 2 \mathrm{mmol} / 1$. 
Tab. 2. Differences found between method 1 and method 3 .

\begin{tabular}{lcc}
\hline & $\begin{array}{l}\text { Relative Differences } \\
\text { of methods } \\
\text { (method 1-method 3) } \cdot 100\end{array}$ & $\begin{array}{l}\text { Absolute differences } \\
\text { of methods }\end{array}$ \\
& \multicolumn{2}{c}{ method 1 } \\
(method 1 - method 3)
\end{tabular}

glucose-6-phosphate dehydrogenase/NAD ${ }^{+}$. It is seen that with the $\mathrm{NAD}^{+}$-system lower values result as compared to NADP ${ }^{+}$. These relative differences of methods are shown in figure 4 as a function of method 1 (yẹașt glucose-6-phosphate dehydrogenase $/ \mathrm{NADP}^{+}$). They are tabulated in table 2.

Relative differences between method 1 and 2 for all - except two values - are $<10 \%$ with a mean value of $-0.8 \%$ only, whereas relative differences between method 1 and 3 scatter widely from -5 to $+20 \%$ with a mean value of $5.8 \%$ higher activity of the yeast glucose6-phosphate dehydrogenase/NADP ${ }^{+}$-syștem. This magnitude of difference in methods ușing NAD ${ }^{+}$compared to methods with $\mathrm{NADP}^{+}$was expected and is attributable to interfering enzymes in serum, e.g. lactate dehydrogenase. We therefore recommend the use of $\mathrm{NADP}^{+}$with either yeast or leuconostoc glucose-6-phosphate dehydrogenase for assays of creatine kinase in serum.

\section{References}

1. Șzász, G., Gruber, W. \& Bernt, E. (1976). Clin. Chem. 22, 650-656.

2. Szász, G., Gerhardt, W., Gruber, W. \& Bernt, E. (1976). Clin. Chem. 22, 1806-1811.

3. Szász, G., Gerhardt, W. \& Gruber, W. (1977). Clin. Chem. 23, 1888-1892.

4. Szász, G. \& Gruber, W. (1978). Clin. Chem. 24, 245-249.

5. Szász, G., Gerhardt, W. \& Gruber, W. (1978). Clin. Chem.

C̈lin. Chem. 24, 1557-1563.

6. Szász, G., Waldenstróm, J. \& Grubẹr, W. (1979). Clin. Chem. $25,446-452$.

7. Recommended method for the determination of creatine kinase in blood. The Committee on Enzymes of the Scandinavian Society for Clinical Chemistry and Clinical Physiology. Scand. J. Clin. Lab. Invest. 36, 711 (1976).

8. Recommendations pour la mesure de l'activite catalytique de la greatine kinase dans le serum humain a $30^{\circ} \mathrm{C}$. Commission "Enzymologie" of the Comite de Standardisation of the Societe Française de Biologie Clinique. Ann. Biol. Clin. 34, 297 (1976).

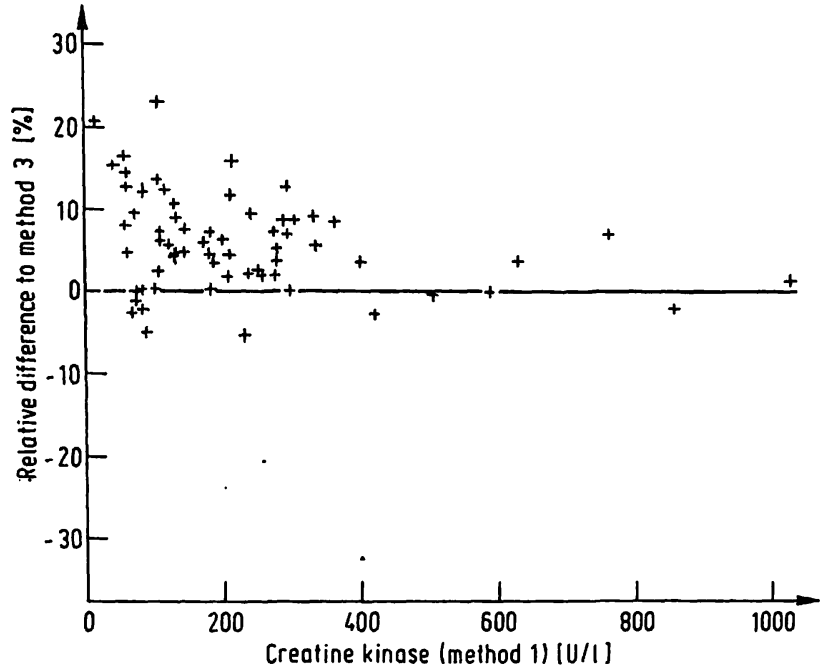

Fig. 4. Relative differences of methods (method 1-method 3) - $100 /$ method 1 shown as related to activities found with method 1.

Method 1: yeast glucose-6-phosphate dehydrogenase $1.73 \mathrm{kU} / \mathrm{l}$ assay volume $\mathrm{NADP}^{+}: 2 \mathrm{mmol} / 1$.

Method 3: leuconostoc glucose-6-phosphate dehydrogenase: $1.73 \mathrm{kU} / \mathrm{l}$ assay volume $\mathrm{NAD}^{+}: 2 \mathrm{mmol} / \mathrm{l}$

\section{Acknowledgement}

The assistance of $S$. Looser in preparing the experiments and of $D$. Markowetz for the summary of results is gratefully acknowledged.

9. Recommendations of the German Society for Clinical Chemistry. Standardization methods for the estimation of enzyme activities in biological fluids. Standard method for the determination of creatine kinase activity. Revised draft of 1976. J. Clin. Chem. Clin. Biochem. 15, 255-260 (1977)

10. Aanbevolen methode voor het meten van de activiteit van kreatine kinase (CK) in serum. Enzymcomissie of the Nederlandse Vereniging voor Klinische Chemie. Mededelingen 3, 220 (1978).

11. Empfohlene Methoden zur Bestimmung von 6 Enzymen im Blutplasma: GOT, GPT, LDH, ALP, CPK und GGT. Fachkommission der Schweizerischen Gesellschaft für Klinische Chemie. Bull. Schweiz. Ges. Klin. Chem. 1978, p. 15.

12. Noldmann, E. A. \& Kuby, S. A. (1963) Enzymes 7, 223-242. 13. De Moss, R. D. (1955) Enzymol. 1. 328-334.

14. Da Fonseca-Wollheim, F. (1973) Z. Klin. Chem. Klin. Biochem. $11,426-431$

15. Szász, G., Busch, E. W., Koller, P. U. \& Vollmar, J. (1976). Clin. Chem. 22, 1213.

16. Gruber, W. (1978) Clin. Chem. 24, 177-178.

Dr. W. Gruber Research Center Boehringer Mannheim GmbH D-8132 Tutzing 


\section{Lieferanten-Nachweis}

- Autoklaven

HEINICKE INSTRUMENTS

Laborgerätebau $\mathrm{GmbH}$

8223 Trostberg. Pf. 1203

Ruf $08621 / 2085$, FS 563110

- Bakteriologie

GTTO NCRDHALD KG

2000 Hamburg 50, Heinrichstr. 5

Ruf 040/4328 27

\section{- Brutschränke}

HEINICKE INSTRUMENTS

Laborgerätebau GmbH

8223 Trostberg. Pf. 1203

Ruf $08621 / 2085$, FS 563110

- Chromatographie-Pumpen

VERDER (DEUTSCHLAND) GMBH 4000 Düsseldorf, Himmeigeisterstr. 60 Ruf $0211 / 334513$

FS: 08585539

- Digitale Verdünnungsgeräte

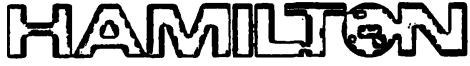

Hamilton Deutschland GmbH Postfach 110427

Otto-Röhm-Str. 74

D.6100 Darmstadt

Ruf 061 51/85085-86, FS 0419684 .

- Digitale Dispensiergeräte

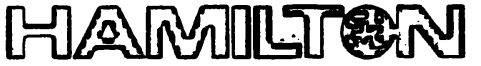

Hamilton Deutschland GmbH

Postfach 110427

Otto-Röhm-Str. 74

D-6100 Darmstadt

Ruf $06151 / 85085-86$, FS 0419684

- Elektrophorese

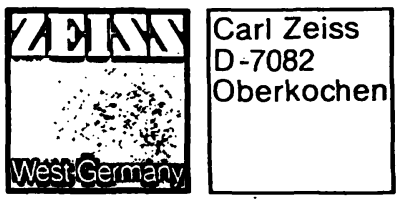

- Filterphotometer

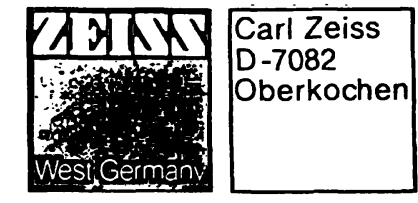

- Flammenphotometer

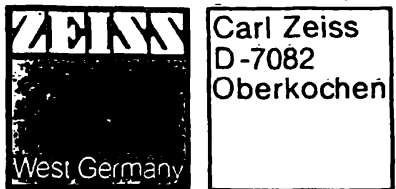

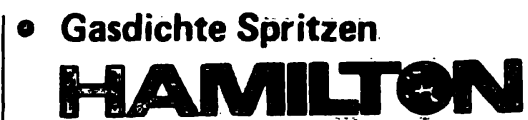

Hamilton Deutschland $\mathrm{GmbH}$

Postfach 110427

Otto-Röhm-Str. 74

D.6100 Darmstadt

Ruf $06151 / 85085-86$, FS 0419684

- Infrarot-Spektralphotometer

\begin{tabular}{|l|l|}
\hline M IXY & $\begin{array}{l}\text { Carl Zeiss } \\
\text { D-7082 } \\
\text { Oberkochen } \\
\text { Wesjigermand }\end{array}$ \\
\hline
\end{tabular}

- Lảborspülmittel

deconex ${ }^{\circledR}$

das umfassende Reinigungspro-

gramm für Labors aller Bereiche

BORER CHEMIE AG

Zürcher Str. 125

Postfach 352

CH-8952 Schlieren-Zürich

Ruf (01) 7301535

Telex CH 54031 SPONA

- Microliterspritzen ${ }^{\circledR}$

\section{HA口国网}

Hamilton Deutschland GmbH

Postfach 110427

Otto-Röhm-Str. 74

D-6100 Darmstadt

Ruf 061 51/850 85-86, FS 0419684

- Mikroskope

HERTEL \& REUSS

3500 Kassel, Quellhofstr. 67

Ruf 0561/8 3006

WILL WETZLAR KG

Optische Werke

Postfach 40

6331 Nauborn-Wetzlär

Ruf $06441 / 23071.4$
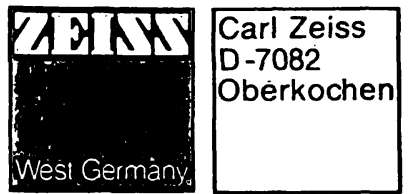

- pH-Meßgeräte +

pH-Glaselektroden

INGOLD pH.Meßtechnik

6000 Frankfurt 1

Postf. 3308, Ruf $0611 / 295301$

- Photometer

WNIXY $\begin{aligned} & \text { Carl Zeiss } \\ & \text { D-7082 } \\ & \text { Oberkochenn }\end{aligned}$
Wesi Germany

- Polarimeter

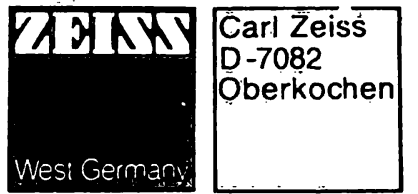

\section{- Radioaktive Stoffe}

AMERSHAM BUCHLER

3300 Braunschweig, Postf. 1120

Ruf $053.07 / 46$ 93-97

\section{- Reinigungsmittel}

deconex ${ }^{(}$

das umfassende Reinigungspro-

gramm für Labors aller Bereiche

BORER CHEMIE AG

Zürcher Str. 125

Postfach 352

CH-8952 Schlieren-Zürich

Ruf (01) 7301535

Telex CH 54031 SPONA

\section{- Spektralphotometer}

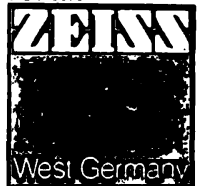

Carl Zeiss'
D-7082
Oberkochen

\section{- Schlauchpumpen}

VERDER (DEUTSCHLAND) GMBH 4000 Düsseldorf, Himmelgeisterstr. 60 Ruf $0211 / 334513$, FS: 08585539

- Sterilisierpapiere

J.C. BINZER

3559 Hatzfeld

Ruf $06467 / 3$ 18, FS: 0482224

- Trockenschränke

HEINICKE INSTRUMENTS

Laborgeràtebàu $\mathrm{GmbH}$

8223 Trostberg, Pf. 1203

Ruf $08621 / 2085$, FS 563110

- Waschautomaten für Laborglas

HEINICKE INSTRUMENTS

Laborgerätebau $\mathrm{GmbH}$

8223 Trostberg, Pf. 1203

Ruf $08621 / 2085$, FS 563110

\section{- Zentrifugen}

BERTHQLD HERMLE KG

7209 Gosheim. Postfacti 1240

Ruf $07426 / 1061$ "? 